\title{
Measuring Cosmological Parameters with Cosmic Microwave Background Experiments
}

\author{
J. Richard Bond, ${ }^{1}$ Robert Crittenden, ${ }^{2}$ Richard L. Davis, ${ }^{2}$ George Efstathiou, ${ }^{3}$ Paul J. \\ Steinhardt ${ }^{2}$ \\ (1) Canadian Institute for Theoretical Astrophysics, University of Toronto, Toronto, Ontario, \\ Canada M5S 1A7 \\ (2) Department of Physics, University of Pennsylvania, Philadelphia, PA 19104 \\ (3) Department of Physics, Oxford University, Oxford, England OX1 3RH
}

\begin{abstract}
The cosmic microwave background anisotropy is sensitive to the slope and amplitude of primordial energy density and gravitational wave fluctuations, the baryon density, the Hubble constant, the cosmological constant, the ionization history, etc. In this Letter, we examine the degree to which these factors can be separately resolved from combined small- and large-angular scale anisotropy observations. We isolate directions of degeneracy in this cosmic parameter space, but note that other cosmic observations can break the degeneracy.
\end{abstract}

PACS NOs: 98.80.Cq, 98.80.Es, 98.70.Vc

Typeset using REVTEX

The observation of large-angular scale $\left(\sim\right.$ large- and small-angular scale $\left(\lesssim 1^{\circ}\right)$ exper$10^{\circ}$ ) fluctuations in the Cosmic Microwave iments [0] are anticipated. In this LetBackground(CMB) [1,2] marks the beginning ter, we explore the degree to which the CMB of a new age of precision measurement in cos- anisotropy observations can determine cosmology [3 [1]. Dramatic improvements in mological parameters such as the slope of the 
initial power spectrum, the age of the universe and the cosmological constant. We find that $\mathrm{CMB}$ anisotropy measurements alone cannot fix the parameters individually; however, a non-trivial combination of them can be determined. More concretely, for models based on the generation of gaussian, adiabatic fluctuations by inflation, we have identified a new variable $\widetilde{n}_{s}$, a function of the basic parameters that can be fixed to great precision by CMB anisotropy observations. Distinct models with nearly the same value of $\widetilde{n}_{s}$ cannot be discriminated by CMB data alone. In a likelihood analysis, this leads to error contours centered around a highly elongated maximum-likelihood surface inside which $\widetilde{n}_{s}$ is approximately constant. However, when combined with other, independent cosmological observations, the determination of $\widetilde{n}_{s}$ is a powerful tool for testing models and measuring fundamental parameters.

We parameterize the space by $\left(C_{2}^{(S, T, I s, \ldots)}, n_{s, t, i s, \ldots}, \mathrm{h}, \Omega_{B}, \Omega_{\Lambda}, \Omega_{C D M}, \Omega_{H D M}\right.$ where $H_{0}=100 \mathrm{~h} \mathrm{~km} \mathrm{sec}^{-1} \mathrm{Mpc}^{-1}$ is the Hubble parameter, and $\Omega_{B, \Lambda, C D M, H D M, \ldots}$ are the energy densities associated with baryons, cosmological constant $(\Lambda)$, cold and hot dark matter, etc., divided by the critical density. We use the CMB quadrupole moments $C_{2}^{(S, T, I s, \ldots)}$ to parameterize the overall amplitudes of energy density (scalar metric), gravitational wave (tensor metric), isocurvature scalar and other primordial fluctuations predicted by the model. We parameterize the shape of the initial (e.g., postinflation) fluctuation spectra in wavenumber $k$ by power law indices $n_{s, t, i s, \ldots}$, defined at time $t_{i}$ by $k^{3}\left\langle\left|(\widetilde{\delta \rho / \rho})\left(k, t_{i}\right)\right|^{2}\right\rangle \propto k^{n_{S}+3}$ and $k^{3}\left\langle\left|\widetilde{h}_{+, \times}\left(k, t_{i}\right)\right|^{2}\right\rangle \propto k^{n_{T}}$, where $\delta \rho / \rho$ and $h_{+, \times}$ are the amplitudes of the energy density and gravitational wave metric fluctuations (for two polarizations), respectively.

In this Letter, we restrict ourselves to subdomains of this large space, in particular to parameters consistent with inflation models of fluctuation generation. Inflation produces a flat universe, hence $\Omega_{C D M}+\Omega_{H D M}+$ $\Omega_{B}+\Omega_{\Lambda} \approx 1$. We also take $\Omega_{H D M}=0$, but note that, for angular scales $\gtrsim 10^{\prime}$, the anisotropy for mixed dark matter models with $\Omega_{C D M}+\Omega_{H D M} \approx 1$ is quite similar to the anisotropy if all of the dark matter is cold. Given $\Omega_{B}$, we impose the nucleosynthesis estimate [1], $\Omega_{B} \mathrm{~h}^{2}=0.0125$, to determine $\mathrm{h}$, but also satisfy the globular cluster and other 
age bounds, [12] $\mathrm{h} \lesssim 0.65$ for $\Omega_{\Lambda}=0$ and $\mathrm{h} \lesssim 0.88$ for $\Omega_{\Lambda} \lesssim 0.6$. (Gravitational lens statistics [13] suggest $\Omega_{\Lambda} \lesssim 0.6$. A straightforward match to galaxy clustering data gives $\Omega_{\Lambda} \leq 1-(0.2 \pm 0.1) \mathrm{h}^{-1}$ if $n_{s} \leq 1$. [14]

Inflation produces adiabatic scalar 15 and tensor 16] Gaussian fluctuations. (For simplicity, we do not consider isocurvature fluctuations [17.) The COBE quadrupole fixes $C_{2}^{(T)}+C_{2}^{(S)}$, but the tensor-to-scalar quadrupole ratio $r \equiv C_{2}^{(T)} / C_{2}^{(S)}$ is undetermined [18]. Inflation does not produce strict power-law spectra, in general, but $n_{s}$ and $n_{t}$ can be defined from power-law best-fits to the theoretical prediction over the scales probed by the CMB. For generic models of inflation, including new, chaotic, and extended models, inflation gives 18,20

$n_{t} \approx n_{s}-1$ and $r \equiv C_{2}^{(T)} / C_{2}^{(S)} \approx 7\left(1-n_{s}\right)$.

Measuring $r$ and $n_{s}$ to determine whether they respect Eq. (1) is a critical test for inflation. With this set of assumptions, we have reduced the parameter-space to three-dimensions, $\left(r \mid n_{s}, \mathrm{~h}, \Omega_{\Lambda}\right)$ (where $\Omega_{B}=$ $0.0125 h^{-2}$ and $\left.\Omega_{C D M}=1-\Omega_{B}-\Omega_{\Lambda}\right)$. We explicitly display both $r$ and $n_{s}$ but with a "|" as a reminder that $r$ is determined by Eq. (1) given $n_{s}$; we have also assumed $n_{t}=n_{s}-1$. Our results are based on numerical integration of the general relativistic Boltzmann, Einstein, and hydrodynamic equations for both scalar [21] and tensor metric fluctuations using methods reported elsewhere 20]. Included in the dynamical evolution are all the relevant components: baryons, photons, dark matter, and massless neutrinos. The temperature anisotropy, $\Delta T / T(\theta, \phi)=\sum_{\ell m} a_{\ell m} Y_{\ell m}(\theta, \phi)$, is computed in terms of scalar and tensor multipole components, $a_{\ell m}^{(S)}$ and $a_{\ell m}^{(T)}$, respectively. For inflation, each multipole for the two modes is predicted to be statistically independent and Gaussian-distributed, fully specified by angular power spectra, $C_{\ell}^{(S)}=\left\langle\left|a_{\ell m}^{(S)}\right|^{2}\right\rangle$ and $C_{\ell}^{(T)}=\left\langle\left|a_{\ell m}^{(T)}\right|^{2}\right\rangle$

Our results are presented in a series of two-panel figures (e.g. see Fig. 1). The upper plots show the spectrum $C_{\ell}$ 's normalized to COBE, and the lower bar charts show the predicted $(\Delta T / T)_{r m s}$ for idealized experiments spanning $10^{\circ}$ to $2^{\prime}$. The bar chart is constructed by computing $\left\langle(\Delta T / T)^{2}\right\rangle=$ $\frac{1}{4 \pi} \sum(2 \ell+1) C_{\ell} W_{\ell}$, where $W_{\ell}$ is a filter function that quantifies experimental sensitivity. 
FIG. 1. Top: Power spectra as a function of multipole moment $\ell$ for $\left(r=0 \mid n_{s}=1\right)$, $\left(r=0.7 \mid n_{s}=0.9\right) \quad$ and $\quad\left(r=1.4 \mid n_{s}=0.8\right) \quad$ where $\mathrm{h}=0.5$ and $\Omega_{\Lambda}=0$ for all models. The spectra in all figures are normalized by the COBE keeping $h=0.5$. In the lower curve, $\Omega_{\Lambda}=0$ but $\sigma_{T}^{2}\left(10^{\circ}\right) \equiv(4 \pi)^{-1} \sum(2 \ell+1) C_{l} \exp (-\ell(\ell+1) / 158.4)$.h is increased from 0.5 to 0.65 (hence $\Omega_{B}$ drops (a Gaussian filter with $10^{\circ}$ fwhm), observed by DMR to be $\sim 1.2 \times 10^{-10}$, with about a $30 \%$ error. Bottom: $(\Delta T / T)_{r m s}$ levels with 1-sigma cosmic variance error bars for nine experiments assuming full-sky coverage. [For $N_{D}=50$ patches and a unity signal-to-noise ratio, the variance is 20\%; see Eq. (2)]. The gaussian coherence angle is indicated below each experiment; see Refs. 1-11 for acronyms.

20,22 Errors arise from experimental noise and "cosmic variance", the latter a theoretical uncertainty due to observing the fluctuation distribution from only one vantage point. The errors bars represent cosmic variance alone assuming full-sky coverage, exemplifying the limiting resolution achievable with CMB experiments. For more realistic error bars, consider a detection obtained from measurements $(\overline{\Delta T / T})_{i} \pm \sigma_{D}$ (where $\sigma_{D}$ represents detector noise) at $i=1, \ldots, N_{D}$ experimental patches sufficiently isolated from
FIG. 2. Power spectra as a function of $\ell$ for scale-invariant models, with $r=0 \mid n_{s}=1$. The middle curve shows $\mathrm{h}=0.5$ and $\Omega_{\Lambda}=0$. In the upper curve, $\Omega_{\Lambda}$ is increased to 0.4 while from 0.5 to 0.3$)$. The spectra are insensitive to changes in h for fixed $\Omega_{B}$. Increasing $\Omega_{\Lambda}$ or $\Omega_{B}$ increases the power at $\ell \sim 200$.

each other to be largely uncorrelated. For large $N_{D}$, the likelihood function falls by $e^{-\nu^{2} / 2}$ from a maximum at $(\Delta T / T)_{\max }$ when $\left(\frac{\Delta T}{T}\right)^{2}=\left(\frac{\Delta T}{T}\right)_{\max }^{2} \pm \sqrt{\frac{2}{N_{D}}} \nu\left[\left(\frac{\Delta T}{T}\right)_{\max }^{2}+\sigma_{D}^{2}\right]$.

An experimental noise $\sigma_{D}$ below $10^{-5}$ is standard now, and a few times $10^{-6}$ is soon achievable, hence if systematic errors and unwanted signals can be eliminated, the 1-sigma $(\nu=1)$ relative uncertainty in $\Delta T / T$ will be from cosmic-variance alone, $1 / \sqrt{2 N_{D}}$, falling below $10 \%$ for $N_{D}>50$. The optimal variance limits shown in the figures roughly correspond to filling the sky with patches separated by $2 \theta_{f w h m}$.

Figure 1 shows a sequence of spectra with varying $r \mid n_{s}$. The characteristic feature is increasingly suppressed small-angular signal as 
FIG. 3. Examples of different cosmologies with nearly identical spectra of multipole moments and $(\Delta T / T)_{r m s}$. The solid curve is $\left(r=0 \mid n_{s}=1, \mathrm{~h}=0.5, \Omega_{\Lambda}=0\right) . \quad$ The other two curves explore degeneracies in the $\left(r=0 \mid n_{s}=1, \mathrm{~h}, \Omega_{\Lambda}\right)$ and $\left(r \mid n_{s}, \mathrm{~h}=0.5, \Omega_{\Lambda}\right)$ planes. In the dashed curve, increasing $\Omega_{\Lambda}$ is almost exactly compensated by increasing h. In the dot-dashed curve, the effect of changing to $r=0.42 \mid n_{s}=0.94$ is nearly compensated by increasing $\Omega_{\Lambda}$ to 0.6 .

$r$ increases and $n_{s}$ decreases. [18,20] Although cosmic variance is significant for large-angle experiments, 23] it can shrink to insignificant levels at smaller scales if large maps are made. It appears that $r \mid n_{s}$ would be experimentally resolvable if $\Lambda, \mathrm{h}$ and ionization history were known.

Figure 2 shows the effects of varying $\Omega_{\Lambda}$ or $H_{0}$ compared to our baseline (solid line) spectrum $\left(r=0 \mid n_{s}=1, \mathrm{~h}=0.5, \Omega_{\Lambda}=0\right)$. Increasing $\Omega_{\Lambda}$ enhances small-angular scale anisotropy by reducing the red shift $z_{\text {eq }}$ at which radiation-matter equality occurs; increasing $\mathrm{h}$ increases $z_{\text {eq }}$ and so has the opposite effect. Increasing $\Omega_{\Lambda}$ also changes slightly the spectral slope for $\ell \lesssim 10$ due to
FIG. 4. Power spectra for models with standard recombination (SR), no recombination $(\mathrm{NR})$, and 'late' reionization $(\mathrm{LR})$ at $z=50$. In all models, $\mathrm{h}=0.5$ and $\Omega_{\Lambda}=0 . \mathrm{NR}$ or reionization at $z \geq 150$ results in substantial suppression at $\ell \geq 100$. Models with reionization at $20 \leq z \leq 150$ give moderate suppression that can mimic decreasing $n_{s}$ or increasing h; e.g., compare the $n_{s}=0.95$ spectrum with SR (thin, dot-dashed) to the $n_{s}=1$ spectrum with reionization at $z=50$ (thick, dot-dashed).

$\Lambda$-suppression of the growth of scalar fluctuations [24]. The bar chart shows that either $r \mid n_{s}, \Omega_{\Lambda}$, or h can be resolved if the other two parameters are known.

A degree of "cosmic confusion" arises, though, if $r \mid n_{s}, \Omega_{\Lambda}$ and $\mathrm{h}$ vary simultaneously. Figure 3 shows our baseline spectrum and spectra for models lying in a twodimensional surface of $\left(r \mid n_{s}, \mathrm{~h}, \Omega_{\Lambda}\right)$ which produce nearly identical spectra. In one case, $r \mid n_{s}$ is fixed, and increasing $\Omega_{\Lambda}$ is nearly compensated by increasing $h$. In the second case, $\mathrm{h}$ is fixed, but increasing $\Omega_{\Lambda}$ is nearly compensated by decreasing $n_{s}$ (with $r$ given by Eq. (1)). 25

Further cosmic confusion arises if we also 
consider ionization history. 26] We expand the parameter-space to include $z_{R}$, the red shift at which we suppose sudden, total reionization of the intergalactic medium. Fig. 4 compares spectra with standard recombination (SR), no recombination (NR) and late reionization $(\mathrm{LR})$ at $z_{R}=50$, where $\mathrm{h}=0.5$ and $\Omega_{\Lambda}=0$. NR represents the behavior if reionization occurs early $\left(z_{R}>>200\right)$. The spectrum is substantially suppressed for $\ell \gtrsim 200$ compared to any SR models. Experiments at $\lesssim 0.5^{\circ}$ scale can clearly identify NR or early reionization $\left(z_{R} \gtrsim 150\right.$ gives qualitatively similar results to NR). Reionization for $20 \lesssim z_{R} \lesssim 150$ results in modest suppression at $\ell \approx 200$, which can be confused with a decrease in $n_{s}$ (see figure).

The results can be epitomized by some simple rules-of-thumb: Over the $30^{\prime}-2^{\circ}$ range, $(\Delta T / T)_{\mathrm{rms}}^{2}$ is roughly proportional to the maximum of $\ell(\ell+1) C_{\ell}$ (the first Doppler peak). Since the maximum (corresponding to $\sim .5^{\circ}$ scales) is normalized to COBE DMR (at $\sim 10^{\circ}$ ), its value is exponentially sensitive to $n_{s}$. Since scalar fluctuations account for the maximum, the maximum decreases as $r$ increases. The maximum is also sensitive to the red shift at matter-radiation equality (or, equivalently, $\left.\left(1-\Omega_{\Lambda}\right) h^{2}\right)$, and to the optical depth at last scattering for late-reionization models, $\sim z_{R}^{3 / 2}$. These observations are the basis of an empirical formula (accurate to $\lesssim 15 \%$ )

$$
\left.\frac{\ell(\ell+1) C_{\ell}}{2 \pi \sigma_{T}^{2}\left(10^{\circ}\right)}\right|_{\max } \approx A e^{B \tilde{n}_{s}}
$$

where $A=0.1, B=3.56$, and

$$
\begin{aligned}
\tilde{n}_{s} \approx & n_{s}-0.28 \log (1+0.8 r) \\
& -0.52\left[\left(1-\Omega_{\Lambda}\right) h^{2}\right]^{\frac{1}{2}}-0.00036 z_{R}^{3 / 2}+.26,
\end{aligned}
$$

where $r$ and $n_{s}$ are related by Eq. (1) for generic inflation models, and $z_{R} \lesssim 150$ is needed to have a local maximum. ( $\tilde{n}_{s}$ has been defined such that $\tilde{n}_{s}=n_{s}$ for $r=0, h=$ $0.5, \Omega_{\Lambda}=0$, and $\left.z_{R}=0.\right)$

Our central result is that CMB anisotropy experiments can determine $\tilde{n}_{s}$, but variations of parameters along the surface of constant $\tilde{n}_{s}$ produce indistinguishable CMB anisotropy. Given present uncertainties in $\mathrm{h}, \Omega_{\Lambda}$ and $z_{R}$, it will be possible to determine the true spectral index $n_{s}$ (or $r$ ) to within $10 \%$ accuracy using the CMB anisotropy alone. Quantitative improvement can be gained by invoking constraints from large-scale structure, e.g., galaxy velocity and cluster distributions, 
although the results are model-dependent. Ultimately, tighter limits on $\Omega_{\Lambda}, \mathrm{h}$, ionization history, and the dark matter density are needed before the CMB anisotropy can develop into a high precision test of inflation (Eq. (1)) and primordial gravitational waves.

This research was supported by the DOE at Penn (DOE-EY-76-C-02-3071), NSERC at Toronto, the SERC at Oxford and the Canadian Institute for Advanced Research.

\section{REFERENCES}

[1] DMR: G.F. Smoot et al, Ap. J. Lett.396, L1 (1992).

[2] K. Ganga et al Astrophys. J. 410, L57 (1993).

[3] MSAM (2-beam): E. S. Cheng et al preprint (1993).

[4] TEN: R.A. Watson et al, Nature 357, 660 (1992).

[5] SP91 (South Pole 1991): T. Gaier et al, Astrophys. J. Lett. 398, L1 (1992); J. Schuster et al, Astrophys. J. Lett 412, L47 (1993).

[6] SK: E.J. Wollack, N.C. Jarosik, C.B. Netterfield, L.A. Page, D. Wilkinson, Princeton preprint (1993).

[7] OVRO: A.C.S. Readhead et al,Astrophys. J. 346 556, (1989).

[8] MAX: P. Meinhold et al, Astrophys. J. Lett. 409, L1 (1993); J. Gunderson et al, Astrophys. J. Lett. 413, L1 (1993).

[9] WD2 (White Dish): G.S. Tucker, G.S. Griffin, H. Nguyen, and J.B. Peterson, Princeton preprint, (1993). 
[10] PYTHON: M. Dragovan et al, private communication.

[11] T.P. Walker, G. Steigman, D.N. Schramm, K.A. Olive, \& H.S. Kang, Astrophys. J 376, 51 (1991).

[12] E.W. Kolb and M. S. Turner, The Early Universe, (Redwood City: AddisonWesley, 1990).

[13] D. Maoz and H.-W. Rix, to appear in Astrophys. J 415 (1993).

[14] e.g., G. Efstathiou, J.R. Bond and S.D.M. White, Mon. Not. R. astr. Soc.258, 1P (1992).

[15] J. Bardeen, P. J. Steinhardt and M. S. Turner, Phys. Rev. D28, 679 (1983); A. H. Guth and S.-Y. Pi, Phys. Rev. Lett. 49, 1110 (1982); A. A. Starobinsky, Phys. Lett. B117, 175 (1982); S. W. Hawking, Phys. Lett. B115, 295 (1982).

[16] L.F. Abbott M. Wise, Nucl. Phys. B 244, 541 (1984); A.A. Starobinsky, Sov. Astron. Lett. 11, 133 (1985).

[17] Structure formation by nearly scaleinvariant isocurvature fluctuations is ruled out, but they could be present as a sub-dominant mode (e.g., J. Bardeen, J.R. Bond and G. Efstathiou, Astrophys. J. 321, 28 (1987)).

[18] R.L. Davis, H.M. Hodges, G.F. Smoot, P.J. Steinhardt, and M.S. Turner, Phys. Rev. Lett. 69, 1856 (1992); see also [20] for discussion of exceptions to Eq (1).

[19] F. Lucchin, S. Matarrese, and S. Mollerach, Astrophys. J.Lett. 401, 49 (1992); D. Salopek Phys. Rev. Lett. 69, 3602 (1992); A. Liddle and D. Lyth, Phys.Lett.B 291, 391 (1992); V. Sahni and T. Souradeep, Mod. Phys. Lett. A7, 3541 (1992); J.E. Lidsey and P. Coles, Mon. Not. Roy. astr. Soc. 258, 57P (1992); F.C. Adams, J.R. Bond, K. Freese, J.A. Frieman and A.V. Olinto, Phys. Rev. D47, 426 (1993).

[20] R.Crittenden,

J.R.Bond, R.L.Davis, G.Efstathiou and P.J.Steinhardt, Phys. Rev. Lett. 71, 324 (1993).

[21] J.R. Bond and G. Efstathiou, Astrophys. J. 285, L45 (1984); Mon. Not. R. astr. Soc. 226, 655-687 (1987).

[22] J.R. Bond, G. Efstathiou, P.M. Lubin, 
and P. Meinhold, Phys. Rev. Lett. 66, 2179 (1991).

[23] M. White, L. Krauss and J. Silk, Yale preprint, YCTP-P44-92.

[24] L. Kofman and A.A. Starobinsky, Sov. Astron. Lett. 11, 271 (1986).

[25] L. Kofman, N. Gnedin and N. Bahcall, Astrophys.J. 413, 1 (1993).

[26] Inflation-based models are likely to have $z_{R}$ low enough to have little effect, 21] but, by imposing it, we can illustrate the qualitative behavior for models which should have $z_{R}$ high (e.g., cosmic strings and textures). 